\title{
Generalized Formulation of Linear Nonquadratic Weighted Optimal Error Shaping Guidance Laws
}

\author{
Namhoon Cho * \\ Agency for Defense Development, Daejeon, 34186, Republic of Korea \\ Youdan $\mathrm{Kim}^{\dagger}$ \\ Seoul National University, Seoul, 08826, Republic of Korea \\ Hyo-Sang Shin $\stackrel{+}{*}$ \\ Cranfield University, Cranfield, Bedfordshire, MK43 OAL, United Kingdom
}

This study presents a novel extension to the theory of optimal guidance laws represented by the non-traditional class of performance indices; non-quadratic-type signal $\mathcal{L}_{p}$-norm for the input weighted by an arbitrary positive function. Various missile guidance problems are generally formulated into a scalar terminal control problem based on the understanding of the predictor-corrector nature. Then, a new approach to derive the optimal feedback law minimising the non-quadratic performance index is proposed by utilising the Hölderian inequality. The proposed extension allows a more general family of formulations for the design of closed-form feedback solutions to various guidance problems to be treated in a unified framework. The equivalence between the proposed approach and other design methodologies is investigated. In general, the type of input norm mainly determines the variability of input during the engagement while trading off against the rate of error convergence. The analytic solution derived in this study is verified by comparison with the solution from numerical optimisation, and the effect of the exponent $p$ in the performance index on trajectory and command is demonstrated by numerical simulations.

\section{Introduction}

Linear Quadratic (LQ) optimal control theory has been the main design methodology for devising missile guidance laws with the needs of shaping the flight trajectory and/or command history in regard to various aspects of the guidance problems. Applications of LQ optimal control theory to missile guidance problems have attracted a substantial amount of previous research efforts in many different directions, such as consideration of terminal constraints [1-6], modulation of trajectory [7-10], integration of guidance and control [11-13], etc. The wide use of the LQ design method is by

\footnotetext{
*Senior Researcher, The 1st R\&D Institute, nhcho91@gmail.com, AIAA Member

${ }^{\dagger}$ Professor, Department of Mechanical and Aerospace Engineering, Institute of Advanced Aerospace Technology, ydkim@snu. ac.kr, AIAA Associate Fellow

$\ddagger$ Professor in Guidance, Navigation and Control, Centre for Autonomous and Cyberphysical Systems, h.shin@cranfield.ac.uk
} 
virtue of its systematic and principled approach of formulating the operational objectives, which is available through the choice of relative weightings in the cost functional quadratic in the state and input variables. Another reason for the LQ design method being widely adopted is that it yields the optimal solutions in the form of state feedback as the outcome of design procedure, which is well-suited to cope with uncertainty or disturbance. These desirable properties facilitated the design of optimal guidance laws with varied problem settings within the LQ regulator framework, particularly in the problem with impact angle constraint [14].

Decades of research on minimum-effort guidance laws have mostly considered performance indices involving the integral square of the lateral acceleration as the measure of cumulative effort. Lateral acceleration arises very naturally as the control input to the system dynamics in various guidance problems because it has a direct relation with the heading rate of the vehicle. As it has been widely known, the physical rationale justifying the choice of a pure quadratic-type performance index is that it leads to the solution minimising loss of speed due to the induced drag since the specific drag depends on lateral manoeuvres according to the quadratic relation.

Nonetheless, the main reason why quadratic norms have been chosen in the design of optimal guidance laws is really mathematical tractability [15]. The same also applies to the reason why the optimality of guidance laws is understood based on LQ inverse optimal control theory $[16,17]$. The classical LQ regulator minimises a quadratic cost subject to linear dynamics with no inequality constraints posed on the state and input variables. Simple analytical solutions are in general known to be no longer available for the problem formulations outside this specific family of optimal control problems.

While most of the trajectory/command shaping guidance laws proposed so far have been based on LQ regulator, the choice of a quadratic-type performance index is not always physically meaningful. The use of quadratic costs might lose its physical meaning when the variable chosen as the input is not the lateral acceleration, when a weighting function on the input is imposed for the purpose of intentional trajectory shaping, or when the costs are the weighted sum of variables in different physical dimensions. The choice of quadratic forms is hardly justified in situations where the vehicle does not undergo drag induced by lateral manoeuvres as in the case of an exoatmospheric interceptor. Also, the $\mathcal{L}_{2}$-norm of input is often referred to as the control energy, but it is not really in the dimension of actual mechanical energy dissipated.

As a matter of fact, a guidance law needs not necessarily be optimal with respect to some metric for precise interception of the target, which is the objective of the foremost priority. Missile guidance problems are usually characterised as the fixed final-state finite-horizon regulation problems with a particular concern on the achieved accuracy of the desired terminal constraints. In this respect, the predictiveness, i.e., predictability of the error in terminal constraints satisfaction, has a higher priority than the optimality. It is widely understood as a consequence that the methodologies for guidance law design basically take the form of predictor-corrector schemes in which the correction input nullifies the predicted final errors. Optimality with respect to a measure can be regarded as an additional benefit 
which is desirable for making the best use of airframe hardware performance limits. The basis for frequent use of optimal control theory in the design of guidance laws also resides in the general understanding that an optimal control is in its essence a predictive control. This is because the solution of a two-point boundary value problem encountered in optimal control design inherently involves the integration of a state equation forward in time.

In these regards, accepting that the optimal control theory is useful in missile guidance, the cost functionals for quantifying the steering effort do not need to be confined only to the weighted quadratic forms. This observation motivates a possible new direction to be addressed in the studies henceforth.

The optimal control of linear system minimising a non-quadratic cost functional has mainly remained as the subject of pure control-theoretical studies and has seldom resulted in practically useful analytical solutions [18-27]. It is desirable to obtain the optimal control law in closed-form in order to take the benefit of feedback and avoid an iterative approach to the two-point boundary value problem. References $[18,19]$ presented solutions to the infinite-horizon minimisation of performance indices containing the sum of positive homogeneous multinomial forms of even degrees in the states and that in the error between states and a given reference, respectively. The efforts for infinite-horizon optimal control with a non-quadratic performance index was reviewed in [20]. In [23], a finite-horizon optimal control problem for a linear time-variant system was discussed under the assumption of convexity of non-quadratic time-variant criteria. However, the formulations in [18-20,23] considered non-quadratic forms for the states while allowing only forms quadratic with respect to the input, the choice which significantly simplifies the derivation of the optimal control law in closed-form. Also, the infinite-horizon minimisation is not of interest in missile guidance. In [21], the design of optimal control for a linear system when the performance index is a convex functional containing strictly convex but not necessarily quadratic function of input was briefly discussed. It was shown that the optimal control law has the form of nonlinear state feedback. However, the result obtained is not a closed-form solution. The focus of [22, 26] was in the approximation of optimal infinite-horizon feedback by receding horizon implementations for the problem with linear dynamics and a non-quadratic cost and in the investigation into the relevant performance bound. Computational dynamic programming recursions to solve finite-horizon problems were presented; that is, no feedback law is provided in closed-form. In [25], the open-loop synthesis problem of the optimal control was addressed for a one-dimensional linear system with a cost functional which is non-quadratic with respect to input and convex with respect to the final state. However, the control law presented in [25] is not in the practically useful closed-loop form. The study of [27] dealt with a linear convex regulator, but again from the theoretical perspective without presenting a closed-form solution.

Previous efforts made in relation to missile guidance scarcely dealt with minimisation of a non-quadratic performance index. The study of [28] is a relevant work on optimal missile guidance that addressed minimisation of a $\mathcal{L}_{q}$-norm allowing $q$ other than 2. It tries to understand the choice of $q$ as the trade-off between minimisation of control energy and minimisation of maximal manoeuvres. However, the analytical solutions are derived only for limited cases of $q$; even numbers. 
The primary purpose of this study is to allow a class of non-quadratic-type cost in problem formulations for the design and analysis of closed-form guidance laws, in an effort to extend the domain of feasible performance index choices and also to overcome the limitations of current theory. To this end, this study proposes a new approach exploiting the Hölder's inequality. The problem formulation considered herein is for the general enough case in which the specific guidance objective of a given mission is represented by the convergence of an appropriate scalar error variable to zero in finite time. In strict mathematical terms, this study generalises and extends linear optimal guidance laws to the minimisers of $\mathcal{L}_{p}$-space input norm for $p \in(1, \infty)$ with arbitrary positive weighting function subject to a real scalar state dynamics and fixed zero final state constraint. As the final result, this study presents a generalised framework for the design of optimal guidance laws with the shaping of error and/or command behaviours through various choices of the input signal norm type and the weighting function.

The original development of this study has its meanings in several respects. From the theoretical point of view, this study mainly contributes to the existing literature by substantially extending the class of problems addressable with analytical design. The extension intended in this study leads to the design of new optimal guidance laws and also provides new understandings of the optimality properties of existing guidance laws. From a practical standpoint, the main contributions of this study are threefold. Firstly, the proposed framework allows the exponent $p$ in the performance index given by a weighted $\mathcal{L}_{p}$-norm of input as an additional design parameter for adjusting the uniformity of total steering effort distribution over time, or in other terms, command variability. The choice of the order of input norm yields another degree-of-freedom which complements the existing approach of shaping the trajectory/command pattern by different choices of the weighting function in the performance index. Secondly, the optimality of the pure proportional navigation guidance law is interpreted with respect to a more general non-quadratic norm. The last is the further generalisation of previous studies on the LQ optimal guidance laws such as those presented in [29, 30]. This study provides a more general and unifying description on the optimal error shaping guidance laws in that the proposed framework encompasses the existing generalised frameworks of LQ optimal guidance laws, e.g., [30] and the references therein, as particular cases obtainable by setting the exponent of the input norm to 2 .

The remainder of this paper is organised as follows: Section II describes a terminal control problem as the formulation identically applicable to various guidance problems from an overviewing perspective and also presents a comprehensive review of existing design methodologies to solve that finite-horizon regulation problem. Section III details a new generalised framework proposed for the design of optimal guidance laws considering a weighted non-quadratic performance index. The properties of the proposed framework and its relation to other design methodologies are discussed in Sec. III along with illustrative examples that show how the proposed framework is connected to the previous studies on optimal error shaping guidance laws. In Sec. IV, numerical simulations are performed to verify the correctness of the theoretical findings of this study and also to demonstrate the effect of the non-quadratic order of input norm on the resulting command and trajectory. Section V summarizes concluding remarks of this study. 


\section{Problem Formulation and Design Methodologies}

In this section, first, the kinematic equations for planar engagement and related variables are briefly summarised in Sec. II.A to facilitate the illustration of the proposed concept with examples in the latter part of this study. In Sec. II.B, a terminal control problem is formulated as a common form of finite-horizon regulator design problem observed in the design of guidance laws. In addition, existing design methodologies that are widely adopted to solve the given finite-horizon regulation problem are introduced in Sec. II.C.

\section{A. Planar Engagement Kinematics}

Figure 1 shows the planar engagement geometry and the notation used for variables. The variables frequently

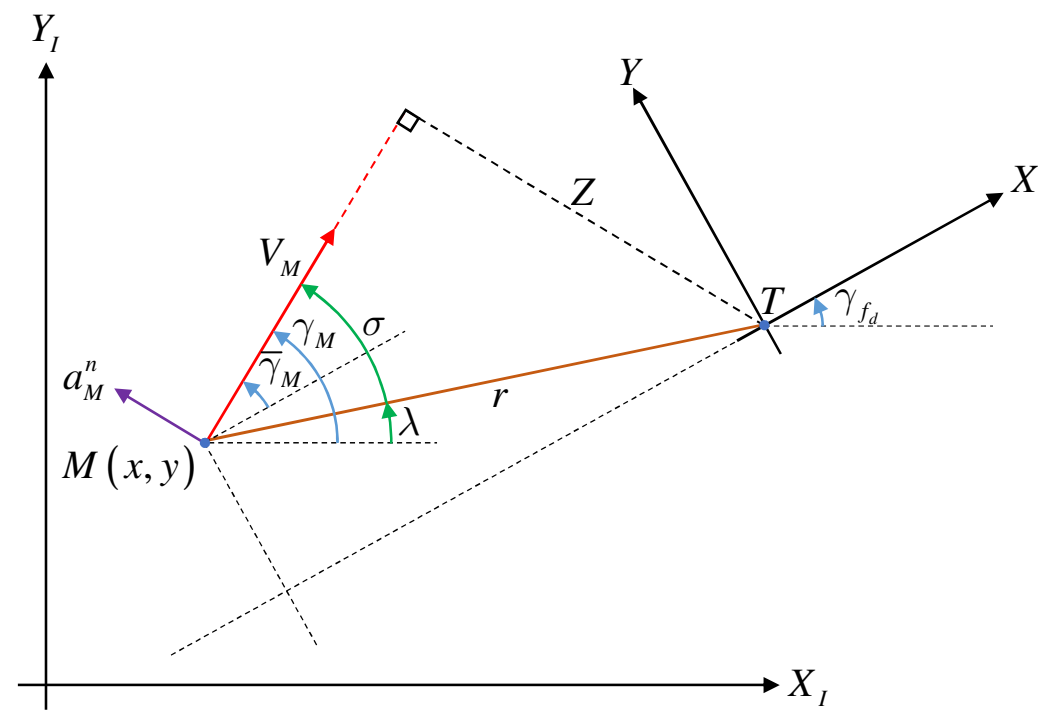

Fig. 1 Planar Engagement Geometry and Notation for Variables

used in the design of a guidance law for impact angle control are depicted in Fig. 1, as the guidance law for impact-angle-constrained interception with the capability of shaping has been one of the most extensively studied subjects of optimal design. Note here that the kinematic equations for stationary target interception are described here with reference to Fig. 1 for the sake of easy explanation in the latter parts of this study, namely, the illustrative examples shown in Secs. III and IV. Nevertheless, the main point of this study does not necessarily confine the type of target motion considered only to the (effectively) stationary one.

In Fig. $1,\left(X_{I}, Y_{I}\right)$ and $(X, Y)$ denote the inertial coordinate system and the impact coordinated system, respectively. The impact coordinate system has its origin at the target $T$, with its $X$-axis aligned with the direction of desired terminal velocity represented by the angle $\gamma_{f_{d}}$ measured positive counter-clockwise from $X_{I}$-axis. For the missile $M,(x, y)$ is the position represented in the impact coordinate system, $V_{M}$ is the speed, $\gamma_{M}$ is the flight path angle, and $a_{M}^{n}$ is the lateral acceleration. Also, $r, \lambda$, and $\sigma$ denote the range, the line-of-sight angle, and the lead angle which is the angle 
between missile's velocity and line-of-sight, respectively.

The motion of the missile can be represented in the impact coordinate system as

$$
\begin{aligned}
\dot{x}=V_{M} \cos \bar{\gamma}_{M}, & x\left(t_{0}\right)=x_{0} \\
\dot{y}=V_{M} \sin \bar{\gamma}_{M}, & y\left(t_{0}\right)=y_{0} \\
\dot{\bar{\gamma}}_{M}=\dot{\gamma}_{M}=\frac{a_{M}^{n}}{V_{M}}, & \bar{\gamma}_{M}\left(t_{0}\right)=\bar{\gamma}_{M_{0}}=\gamma_{M_{0}}-\gamma_{f_{d}}
\end{aligned}
$$

where $\bar{\gamma}_{M} \triangleq \gamma_{M}-\gamma_{f_{d}}$ is the flight path angle error and ()$=\frac{d}{d t}()$. Also, the motion of the missile can be represented in terms of polar coordinate variables as follows:

$$
\begin{aligned}
& \dot{r}=-V_{M} \cos \sigma, \quad r\left(t_{0}\right)=r_{0} \\
& \dot{\lambda}=-\frac{V_{M} \sin \sigma}{r}, \quad \lambda\left(t_{0}\right)=\lambda_{0}
\end{aligned}
$$

where $\sigma \triangleq \gamma_{M}-\lambda$. Equations (1) and (2) together form the basic set of expressions describing the planar engagement.

\section{B. Formulation of Guidance Problems}

In general, the process of guidance law design can typically be framed into two distinct steps: i) definition of error and derivation of its dynamics, and ii) design of input for error regulation.

The first step is the definition of a proper error variable representing the specific objective of each mission, which implicitly entails the formulation of collision condition along with the choice of an independent variable and an input for the error dynamics. The operational objectives of a missile guidance mission include nullification of some form of miss distance or instantaneous heading error, satisfaction of additional final-time constraints on the direction of incidence, flight time, or both at the same time, etc.

By following the notion of predictor-corrector nature which underlies virtually every missile guidance problem, the error is usually defined with respect to the final value of a quantity of interest predicted up to the moment defined as the completion of the engagement. This prediction step requires models of motion for both the missile and the target to be taken as the baseline utilisable for obtaining the uncorrected trajectory, i.e., the trajectory predicted in the absence of missile's correction effort, either in closed-form or in partially closed-form. The prediction model varies depending on the choices for each of its constituents such as assumptions on speed variation and motion pattern of each player [31], the way of considering the environmental component like gravitational acceleration [32], and whether to subsume a baseline guidance law as a nominal manoeuvre strategy.

The meaning of zero correction effort needs careful attention in the case where a baseline guidance law is included as the nominal input. Suppose that the guidance input $u$ consists of the baseline guidance law $u_{\text {base }}$ and the correction input 
$u_{\text {corr }}$, i.e., $u=u_{\text {base }}+u_{\text {corr }}$. The uncorrected trajectory in this case refers to the trajectory predicted from the closed-loop system dynamics without correction input $u_{\text {corr }}$ augmented to $u_{\text {base }}$. In other words, the prediction model becomes the associated closed-loop system dynamics with $u_{\text {corr }} \equiv 0$. Use of a baseline guidance law is an approach widely adopted to the guidance problems with additional terminal constraints beyond interception such as impact angle, impact time, or both of them. In this approach, the uncorrected trajectory guarantees interception of target, and therefore the design of a correction input $u_{\text {corr }}$ can be performed solely to account for zeroing the error related to the additional constraints.

Let $z$ be the performance output variable pertaining to the specified operational objective and $\xi$ represents the independent variable which is in monotone relation with respect to time. Also, let the input $u$ stand for the additional correction input $u_{\text {corr }}$ henceforth if a baseline input $u_{\text {base }}$ is already introduced in the prediction model, unless otherwise stated. Suppose that the evolution of $z$ over $\xi$ is described by a differential equation, i.e.,

$$
\frac{d z}{d \xi}=f(\xi, z, u ; \boldsymbol{\rho})
$$

where $\rho$ denotes all other intermediate variables or parameters. The forward-predictive estimate for the uncorrected trajectory can be obtained at each instant as

$$
\left.\hat{z}\left(\xi_{1}\right)\right|_{u=0} \triangleq \int_{\xi}^{\xi_{1}} f(\tau, z, u=0 ; \boldsymbol{\rho}) d \tau+z(\xi)
$$

where $\xi \triangleq \xi(t)$ and $\xi_{1} \triangleq \xi\left(t_{1}\right)$ for some $t_{1}>t$. The error can be defined by the difference between the instantaneous predicted final value of uncorrected $z$ and the desired final value $z_{f_{d}}$ as

$$
\begin{aligned}
e & \left.\triangleq \hat{z}\left(\xi_{f}\right)\right|_{u=0}-z_{f_{d}} \\
& =\left[\int_{\xi}^{\xi_{f}} f(\tau, z, 0 ; \boldsymbol{\rho}) d \tau+z(\xi)\right]-z_{f_{d}}
\end{aligned}
$$

where $\xi_{f} \triangleq \xi\left(t_{f}\right)$. The desired value $z_{f_{d}}$ in Eq. (5) is usually a constant, and therefore, the error dynamics can be readily derived from Eqs. (3) and (5) as follows:

$$
\frac{d e}{d \xi}=-f(\xi, z, 0 ; \boldsymbol{\rho})+f(\xi, z, u ; \boldsymbol{\rho})
$$

It is noticeable from Eq. (6) that $u$ is responsible for the regulation of $e$ and that the error dynamics will simply be linear in $u$ if the function $f$ is linear in $u$.

Investigations on the error and its dynamics as defined in Eqs. (5) and (6) suggest that the guidance design problems addressed in many earlier studies can reasonably be described in a unified form without much loss of generality. In this regard, the guidance design problem considered in this study is defined as the following single-input single-output 
finite-horizon regulation problem, assuming that both the error and input are scalar variables.

\section{Definition 1 (Guidance Design Problem).}

Let $\xi \in \mathbb{R}, e \in \mathbb{R}$, and $u \in \mathbb{R}$ be the independent variable, error, and input, respectively. Consider the error dynamics given by

$$
e^{\prime}(\xi)=a(\xi) e(\xi)+b(\xi) u(\xi)+c(\xi)
$$

where ()$^{\prime} \triangleq \frac{d}{d \xi}()$ and $b(\xi) \neq 0$ for all $\xi \in\left(\xi_{0}, \xi_{f}\right)$, which renders the system to be controllable. The problem is to design an input $u$ in order to achieve following condition.

$$
e\left(\xi_{f}\right)=0
$$

Note that the presence of forcing term $c(\xi)$ in Eq. (7) will be considered only for the comprehensiveness of the review of existing methods in Sec. II.C, but not for the main development presented in Sec. III.

Remark 1 (Relation Between Error Variable and Operational Objective).

If the error variable $e$ is given by the Zero-Effort-Miss (ZEM), impact angle error, impact time error, heading error, then the result of design will be the Augmented Proportional Navigation Guidance law (APNG), Impact Angle Control Guidance law (IACG), Impact Time Control Guidance law (ITCG), Guidance to Collision law (G2C), respectively. Composite guidance laws for multiple mission objectives might be derived by applying the formulation principle for each objective in a successive manner as in [33]. Reference [30] provided a nice review of guidance laws from the same finite-horizon error regulation perspective.

Remark 2 (Independent Variable of Error Dynamics).

The notation $\xi$ is used for the independent variable of the error dynamics instead of the usual choice of time $t$ for the problem formulation to generally capture many different error shaping guidance problems in a unified manner. The problem formulation of this study considers the independent variable $\xi$ being monotonic with respect to time. The examples for the independent variable of the error dynamics found in missile guidance literature include the time $t$, the range $r$, the arc-length of missile's path $s$, and the downrange-to-go $x_{g o}=-x$.

\section{Existing Design Methodologies}

The next step in the design process is to solve the terminal control problem in Definition 1 . The methods that have been widely adopted for this purpose are discussed in this section. Existing design methodologies can be categorised into three different but interconnected approaches; i) LQ optimal control, ii) input linear parametrisation, and iii) dynamic inversion. They show certain relations to each other, though each approach originates from a different starting point. 


\section{Approach 1: LQ Optimal Control}

Many earlier studies were based on the LQ regulator framework concerning optimality with respect to weighted quadratic performance indices. The LQ optimal terminal control problem for a scalar system is given by

$$
\begin{aligned}
& \text { minimise } \quad J=\int_{\xi}^{\xi_{f}}\left[\frac{1}{2} Q(\tau) e^{2}(\tau)+N(\tau) e(\tau) u(\tau)+\frac{1}{2} R(\tau) u^{2}(\tau)\right] d \tau \\
& \text { subject to } \quad e^{\prime}(\xi)=a(\xi) e(\xi)+b(\xi) u(\xi)+c(\xi) \\
& e\left(\xi_{f}\right)=0
\end{aligned}
$$

where $Q(\xi) \geq 0$ and $R(\xi)>0$ for all $\xi \in\left[\xi_{0}, \xi_{f}\right]$. The optimal control law can be derived as follows [34, 35]:

$$
\begin{aligned}
u^{*}(\xi) & =-R^{-1}(N+b \bar{S}) e(\xi)-R^{-1} b\left(F G^{-1} G_{\Delta}+F_{\Delta}\right) \\
& \triangleq-K_{1}(\xi) e(\xi)+\mu_{1}(\xi)
\end{aligned}
$$

where

$$
\begin{array}{rlrl}
\bar{S} & =S-F^{2} G^{-1} & \\
\dot{S} & =-2 a S-Q+R^{-1}(N+b S)^{2}, & S\left(\xi_{f}\right) & =0 \\
\dot{F} & =-\left[a-(N+b S) R^{-1} b\right] F, & F\left(\xi_{f}\right) & =1 \\
\dot{G} & =R^{-1}(b F)^{2}, & G\left(\xi_{f}\right) & =0 \\
\dot{F}_{\Delta} & =-\left[a-(N+b S) R^{-1} b\right] F_{\Delta}-S c, & F_{\Delta}\left(\xi_{f}\right) & =0 \\
\dot{G}_{\Delta} & =-R^{-1} b^{2} F F_{\Delta}+F c, & G_{\Delta}\left(\xi_{f}\right) & =0
\end{array}
$$

The solution generally requires numerical backward integration. In addition, the closed-form solution for the particular case with $Q(\xi)=N(\xi)=0$ can be derived by using the Schwarz's inequality-based approach as in [30,36], and the result is equivalent to that obtained by simply substituting $Q=N=0$ in Eq. (10).

\section{Approach 2: Input Linear Parametrisation}

Another approach is based on parametrising input or state as linear combination of basis functions [37, 38]. In this approach, the design begins by parametrising the input as

$$
u(\xi)=\alpha \phi(\xi)
$$

where $\phi(\xi)$ is the basis function and $\alpha$ is the constant coefficient that needs to be determined by a boundary condition. As summarised in Sec. A, the solution for the system of Eq. (7) with inputs given by the form of Eq. (12) in the interval 
from $\xi$ to $\xi_{f}$ can be expressed as

$$
e\left(\xi_{f}\right)=\Phi\left(\xi_{f}, \xi\right) e(\xi)+\int_{\xi}^{\xi_{f}} \Phi\left(\xi_{f}, \tau\right)[b(\tau) \alpha \phi(\tau)+c(\tau)] d \tau
$$

where $\Phi\left(\xi_{1}, \xi_{2}\right)$ describes the state transition from $\xi_{1}$ to $\xi_{2}$. The coefficient can be determined by considering the boundary condition of Eq. (8) in Eq. (13) as

$$
\alpha=-\left[\int_{\xi}^{\xi_{f}} \Phi\left(\xi_{f}, \tau\right) b(\tau) \phi(\tau) d \tau\right]^{-1}\left[\Phi\left(\xi_{f}, \xi\right) e(\xi)+\int_{\xi}^{\xi_{f}} \Phi\left(\xi_{f}, \tau\right) c(\tau) d \tau\right]
$$

Finally, the control law can be derived by substituting Eq. (14) in Eq. (12) as follows:

$$
\begin{aligned}
u(\xi)= & -\phi(\xi)\left[\int_{\xi}^{\xi_{f}} \Phi\left(\xi_{f}, \tau\right) b(\tau) \phi(\tau) d \tau\right]^{-1} \Phi\left(\xi_{f}, \xi\right) e(\xi) \\
& \left.-\phi(\xi)\left[\int_{\xi}^{\xi_{f}} \Phi\left(\xi_{f}, \tau\right) b(\tau) \phi(\tau) d \tau\right]\right]_{\xi}^{-1} \int_{\xi}^{\xi_{f}} \Phi\left(\xi_{f}, \tau\right) c(\tau) d \tau \\
\triangleq & -K_{2}(\xi) e(\xi)+\mu_{2}(\xi)
\end{aligned}
$$

Given that the choices in formulation stage already brought the problem to the form described in Definition 1, shaping of the trajectory/command pattern can be performed by different choices of the basis function $\phi(\xi)$. Note that the philosophy of this approach is in essence similar to the interpolation of the current and final points.

\section{Approach 3: Dynamic Inversion}

Shaping guidance laws can also be designed by prescription of the error $e$ as a desired function of $\xi$ satisfying the final constraint, or equivalently, by designating desired closed-loop error dynamics. The design procedure presented in [29] is a notable example of this approach.

Let the desired error profile is given by $e(\xi)=f_{\text {des }}(\xi)$ where $f_{\text {des }}$ is a function which satisfies $f_{\text {des }}\left(\xi_{f}\right)=0$ in light of the condition in Eq. (8). In this case, the input can be obtained by first equating the derivative of desired error profile with the error dynamics in Eq. (7) and then by inversion as

$$
u(\xi)=b^{-1}(\xi)\left[f_{\text {des }}{ }^{\prime}(\xi)-a(\xi) e(\xi)-c(\xi)\right]
$$

If the desired closed-loop error dynamics is specified instead of the error profile itself as $e^{\prime}(\xi)+p_{\text {des }}(\xi) e(\xi)=$ $r_{d e s}(\xi)$, the input can be derived by the dynamic inversion principle as

$$
u(\xi)=b^{-1}(\xi)\left[-\left\{p_{\text {des }}(\xi)+a(\xi)\right\} e(\xi)+\left\{r_{\text {des }}(\xi)-c(\xi)\right\}\right]
$$


Remark 3 clarifies the condition that should be satisfied by the desired closed-loop error dynamics to have zero final error.

Remark 3 (Requirement for First-Order Linear Closed-Loop Error Dynamics). A class of desired error profiles can be characterized by desired error dynamics of the following form.

$$
e^{\prime}(\xi)+p_{\text {des }}(\xi) e(\xi)=r_{\text {des }}(\xi)
$$

For this class of desired error profiles, transient performance characteristics in terms of natural frequency and damping ratio can be speculated from the derivative of Eq. (18) adopting the notion of frozen-time analysis. This way of approximation is not always reasonable, and therefore, careful examination is necessary.

By following the derivation shown in Appendix A, the general solution of Eq. (18) can be expressed as

$$
e(\xi)=F(\xi)\left[\int_{\xi_{0}}^{\xi} F^{-1}(\tau) r_{d e s}(\tau) d \tau+e\left(\xi_{0}\right)\right]
$$

where

$$
F(\xi) \triangleq \exp (h(\xi)) \quad \text { with } h(\xi) \triangleq-\int_{\xi_{0}}^{\xi} p_{\text {des }}(s) d s
$$

For the solution given by Eq. (19) to satisfy the requirement $e\left(\xi_{f}\right)=0$ for any $e\left(\xi_{0}\right)$ and any $r_{\text {des }}(\xi), F\left(\xi_{f}\right)=0$ should be satisfied. This condition can be met only with particular choices of the function $p_{\text {des }}(\xi)$.

The range of the function $F(\xi)$ can include zero if and only if $h(\xi)$ contains $\ln f(\xi)$ as a distinguished term, where $f(\xi)$ is a function satisfying $f\left(\xi_{f}\right)=0$. Otherwise, $F(\xi)$ is strictly greater than zero for every $\xi$. This is because $F(\xi)$ is an exponential function of $h(\xi)$. In other words, if

$$
h(\xi)=\ln f(\xi)+g(\xi)
$$

with $f\left(\xi_{f}\right)=0$, then

$$
F(\xi)=\exp (\ln f(\xi)+g(\xi))=f(\xi) \exp (g(\xi))
$$

and therefore $F\left(\xi_{f}\right)$ becomes zero. Considering the relation defined in Eq. (20), the function $p$ ( $\xi$ ) that corresponds to Eq. (21) can be written as

$$
p_{\text {des }}(\xi)=-h^{\prime}(\xi)=-\frac{f^{\prime}(\xi)}{f(\xi)}-g^{\prime}(\xi)
$$

This observation supports that $p_{\text {des }}(\xi)$ in the desired error dynamics should include a fractional term $\frac{f^{\prime}(\xi)}{f(\xi)}$ with $f(\xi)$ predetermined as the one satisfying $f\left(\xi_{f}\right)=0$. The functions $f(\xi), g(\xi)$, and $r_{\text {des }}(\xi)$ will determine the desired error profile $e(\xi)$. Designers can shape the desired error pattern by adjusting those functions. 


\section{Main Results and Illustrative Examples}

This section is devoted to the main novelty of this study. Section III.A presents the design of optimal guidance command for minimising the $\mathcal{L}_{p}$-norm of input weighted by a general positive function. In Sec. III.B, two notable examples are presented to illustrate the proposed concept from the practical standpoint. It is then followed by discussions on the solution properties and on the connections between the proposed framework and existing methodologies in Sec. III.C.

\section{A. Generalised Weighted Linear Non-quadratic Optimal Guidance}

The proposed framework deals with minimization of weighted $\mathcal{L}_{p}$-norm of input. The design problem considered herein can be described as follows:

$$
\begin{array}{rlrl} 
& & & \\
\text { minimise } & =\int_{\xi}^{\xi_{f}} W(\tau)|u(\tau)|^{p} d \tau \\
\text { subject to } & e^{\prime}(\xi) & =a(\xi) e(\xi)+b(\xi) u(\xi) \\
& e\left(\xi_{f}\right)=0
\end{array}
$$

where $W(\xi)>0$ for all $\xi$ and $p \in(1, \infty)$, and $b(\xi) \neq 0$ for all $\xi \in\left(\xi_{0}, \xi_{f}\right)$. Integration of the given system dynamics from $\xi$ to $\xi_{f}$ gives

$$
e\left(\xi_{f}\right)=\exp \left(\int_{\xi}^{\xi_{f}} a(s) d s\right) e(\xi)+\int_{\xi}^{\xi_{f}} \exp \left(\int_{\tau}^{\xi_{f}} a(s) d s\right) b(\tau) u(\tau) d \tau
$$

Let $\Phi(\alpha, \beta) \triangleq \exp \left(\int_{\beta}^{\alpha} a(s) d s\right)$. Imposing the final error constraint in Eq. (25) yields

$$
\Phi\left(\xi_{f}, \xi\right) e(\xi)=-\int_{\xi}^{\xi_{f}} \Phi\left(\xi_{f}, \tau\right) b(\tau) u(\tau) d \tau
$$

Applying triangle inequality to the preceding equation gives

$$
\left|\Phi\left(\xi_{f}, \xi\right) e(\xi)\right|=\left|\int_{\xi}^{\xi_{f}} \Phi\left(\xi_{f}, \tau\right) b(\tau) u(\tau) d \tau\right| \leq \int_{\xi}^{\xi_{f}}\left|\Phi\left(\xi_{f}, \tau\right) b(\tau) u(\tau)\right| d \tau
$$

Note that the equality holds if and only if $\Phi\left(\xi_{f}, \tau\right) b(\tau) u(\tau)$ does not change its sign in the interval $\left[\xi, \xi_{f}\right]$. The RHS of Eq. (27) can be rewritten by introducing $W(t)$ as a slack variable.

$$
\int_{\xi}^{\xi_{f}}\left|\Phi\left(\xi_{f}, \tau\right) b(\tau) u(\tau)\right| d \tau=\int_{\xi}^{\xi_{f}}\left(\left|\Phi\left(\xi_{f}, \tau\right) b(\tau)\right| W^{-\frac{1}{p}}(\tau)\right)\left(W^{\frac{1}{p}}(\tau)|u(\tau)|\right) d \tau
$$


Applying Hölder's inequality in Lemma 1 (see Appendix B) to Eq. (28) gives

$$
\int_{\xi}^{\xi_{f}}\left|\Phi\left(\xi_{f}, \tau\right) b(\tau) u(\tau)\right| d \tau \leq\left[\int_{\xi}^{\xi_{f}} W(\tau)|u(\tau)|^{p} d \tau\right]^{\frac{1}{p}}\left[\int_{\xi}^{\xi_{f}}\left|\Phi\left(\xi_{f}, \tau\right) b(\tau)\right|^{\frac{p}{p-1}} W^{-\frac{1}{p-1}}(\tau) d \tau\right]^{\frac{p-1}{p}}
$$

Equality in Eq. (29) holds if and only if $\left|\Phi\left(\xi_{f}, \tau\right) b(\tau)\right| W^{-\frac{1}{p}}(\tau)=c_{1} W^{\frac{p-1}{p}}(\tau)|u(\tau)|^{p-1}$ for some $c_{1}$. From the inequality relations shown in Eqs. (27) and (29), we have

$$
\left[\int_{\xi}^{\xi_{f}} W(\tau)|u(\tau)|^{p} d \tau\right]^{\frac{1}{p}} \geq \frac{\left|\Phi\left(\xi_{f}, \xi\right) e(\xi)\right|}{\left[\int_{\xi}^{\xi_{f}}\left|\Phi\left(\xi_{f}, \tau\right) b(\tau)\right|^{\frac{p}{p-1}} W^{-\frac{1}{p-1}}(\tau) d \tau\right]^{\frac{p-1}{p}}}
$$

Equation (30) gives a lower bound on the performance index, and the equality holds if and only if $\Phi\left(\xi_{f}, \tau\right) b(\tau) u(\tau)$ is either non-negative or non-positive for all $\tau \in\left[\xi, \xi_{f}\right]$ and $|u(\tau)|=c_{2}\left|\Phi\left(\xi_{f}, \tau\right) b(\tau)\right|^{\frac{1}{p-1}} W^{-\frac{1}{p-1}}(\tau)$ for some $c_{2}$. By definition, $\Phi\left(\xi_{f}, \tau\right) \geq 0$ for all $\tau \in\left[\xi_{0}, \xi_{f}\right]$ and $b(\tau) \neq 0$ for all $\tau \in\left(\xi_{0}, \xi_{f}\right)$. The function $b(\tau)$ being non-zero during the entire engagement period, $\left(\xi_{0}, \xi_{f}\right)$, implies that there is no point in the interval at which $b(\tau)$ crosses zero and changes its sign. Therefore, the sign of $\Phi\left(\xi_{f}, \tau\right) b(\tau) u(\tau)$ depends only on $u(\tau)$. Considering that $W(\tau)>0$ for every $\tau$, the input should have the following form for the equality in Eq. (30) to hold

$$
u(\tau)=P\left|\Phi\left(\xi_{f}, \tau\right) b(\tau)\right|^{\frac{1}{p-1}} W^{-\frac{1}{p-1}}(\tau)
$$

where $P$ is a constant. Note that $u(\tau)$ of Eq. (31) does not change its sign indeed.

Substitution of Eq. (31) into Eq. (26) results in

$$
\Phi\left(\xi_{f}, \xi\right) e(\xi)=-\int_{\xi}^{\xi_{f}} \Phi\left(\xi_{f}, \tau\right) b(\tau) P \mid \Phi\left(\xi_{f}, \tau\right) b(\tau)^{\frac{1}{p-1}} W^{-\frac{1}{p-1}}(\tau) d \tau
$$

Solving Eq. (32) for $P$ gives

$$
P=-\frac{\Phi\left(\xi_{f}, \xi\right) e(\xi)}{\int_{\xi}^{\xi_{f}} \Phi\left(\xi_{f}, \tau\right) b(\tau)\left|\Phi\left(\xi_{f}, \tau\right) b(\tau)\right|^{\frac{1}{p-1}} W^{-\frac{1}{p-1}}(\tau) d \tau}
$$

Substituting Eq. (33) into Eq. (31) and evaluating at $\tau=\xi$ gives the optimal input in a feedback-form as

$$
\begin{aligned}
u(\xi) & =-\frac{\Phi\left(\xi_{f}, \xi\right)\left|\Phi\left(\xi_{f}, \xi\right) b(\xi)\right|^{\frac{1}{p-1}} W^{-\frac{1}{p-1}}(\xi)}{\int_{\xi}^{\xi_{f}} \Phi\left(\xi_{f}, \tau\right) b(\tau)\left|\Phi\left(\xi_{f}, \tau\right) b(\tau)\right|^{\frac{1}{p-1}} W^{-\frac{1}{p-1}}(\tau) d \tau} e(\xi) \\
& \triangleq-K^{*}(\xi) e(\xi)
\end{aligned}
$$

The final result, Eq. (34), is the linear optimal control law that minimises the weighted non-quadratic performance 
index. Note that the derivation presented above assumed $\frac{d \xi}{d t}>0$ so that $\xi_{f}>\xi$, however, one will arrive at the same final optimal solution by applying the similar procedure even if $\frac{d \xi}{d t}<0$ is supposed.

Remark 4 (Equivalence Between Weighted $\mathcal{L}_{2}$-Norm Minimisation and Pure $\mathcal{L}_{p}$-Norm Minimisation).

For the special cases where $a(\xi)=0$, we have $\Phi\left(\xi_{f}, \tau\right)=1$, and the optimal solution of Eq. (34) can be rewritten as

$$
u(\xi ; p, W(\xi))=-\frac{|b(\xi)|^{\frac{1}{p-1}} W^{-\frac{1}{p-1}}(\xi)}{\int_{\xi}^{\xi_{f}} b(\tau)|b(\tau)|^{\frac{1}{p-1}} W^{-\frac{1}{p-1}}(\tau) d \tau} e(\xi)
$$

An interesting observation made from Eq. (35) is that the optimal solution for $p=2$ with the particular choice of weighting function given by $W(\xi)=|b(\xi)|^{1-\frac{1}{q-1}}$ for some $q \in(1, \infty)$ is identical to the optimal solution for $p=q$ with $W(\xi)=1$, i.e., $u\left(\xi ; 2,|b(\xi)|^{\frac{q-2}{q-1}}\right) \equiv u(\xi ; q, 1)$. That is, a guidance law can be interpreted as the minimiser of weighted $\mathcal{L}_{2}$-norm with the specific weighting function choice as well as the minimiser of pure $\mathcal{L}_{p}$-norm.

\section{B. Illustrative Examples}

The examples presented below clearly show how the proposed framework is related to the earlier studies.

\section{Example 1: Reduction to Linear Quadratic Optimal Case}

Let $\xi=t, a(\xi)=0$, and $p=2$. Because $\Phi\left(\xi_{f}, \tau\right)=1$ with $a(\xi)=0$, the optimal control input for this case becomes

$$
u(t)=-\frac{|b(t)| W^{-1}(t)}{\int_{t}^{t_{f}} b(\tau)|b(\tau)| W^{-1}(\tau) d \tau} e(t)
$$

The function $b(t)$ does not change its sign by definition, and therefore, Eq. (36) reduces to

$$
u(t)=-\frac{b(t) W^{-1}(t)}{\int_{t}^{t_{f}} b^{2}(\tau) W^{-1}(\tau) d \tau} e(t)
$$

and it is known to be the minimiser of $J=\int_{t}^{t_{f}} W(\tau) u^{2}(\tau) d \tau$ with the guarantee of finite-time convergence of $e$ at $t_{f}$. This result is exactly same as the recent work presented in [30]. In this respect, the previous work can be understood as a particular case of the proposed framework. Also, various guidance laws presented in [30] and the references therein, e.g., ZEM shaping guidance, APNG, IACG, ITCG, G2C, etc., can be easily extended to the minimisers of some non-quadratic performance indices by applying the method proposed in this study. Moreover, the discussions on the properties of the LQ optimal solutions apply also to the linear non-quadratic optimal guidance laws in a similar manner.

\section{Example 2: $\mathcal{L}_{p}$-Optimal Pure Proportional Navigation Guidance}

Let us consider a simple example of stationary target interception on a plane, and let $Z$ denote the zero-effort-miss in Fig. 1. Let $\xi=r, e=Z=r \sin \left(\gamma_{M}-\lambda\right)$, and $u=a_{M}^{n}$. The speed of the missile $V_{M}$ is assumed to be constant 
during the entire engagement. For this choice of variables, the system dynamics can be represented with $a(r)=0$, $b(r)=-\frac{r}{V_{M}{ }^{2}}$, and $c(r)=0$, by using Eqs. (1)-(2). It is widely accepted that nullifying $Z$ at the terminal instance leads to successful interception. The weighted $\mathcal{L}_{p}$-optimal control input for this problem can be given as

$$
\begin{aligned}
a_{M}^{n}(r) & =-\frac{\left|-\frac{r}{V_{M}^{2}}\right|^{\frac{1}{p-1}} W^{-\frac{1}{p-1}}(r)}{\int_{r}^{0}-\frac{r}{V_{M}^{2}}\left|-\frac{r}{V_{M}^{2}}\right|^{\frac{1}{p-1}} W^{-\frac{1}{p-1}}(r) d r} Z(r) \\
& =-\frac{r^{\frac{1}{p-1}} W^{-\frac{1}{p-1}}(r)}{\int_{0}^{r} r^{\frac{p}{p-1}} W^{-\frac{1}{p-1}}(r) d r} V_{M}^{2} Z(r)
\end{aligned}
$$

From Eq. (2), we have $\dot{\lambda}=-\frac{V_{M}}{r^{2}} Z$. Substituting $Z=-\frac{r^{2}}{V_{M}} \dot{\lambda}$ into Eq. (38) yields the guidance command expressed in terms of line-of-sight rate.

$$
a_{M}^{n}(r)=\frac{r^{\frac{2 p-1}{p-1}} W^{-\frac{1}{p-1}}(r)}{\int_{0}^{r} r^{\frac{p}{p-1}} W^{-\frac{1}{p-1}}(r) d r} V_{M} \dot{\lambda} \triangleq G(r) V_{M} \dot{\lambda}
$$

This result generalises the quadratic optimality of Pure Proportional Navigation Guidance law (PPNG) presented in [16] to the non-quadratic case. Indeed, substitution of $p=2$ into Eq. (39) gives the same result as shown in the previous work. In addition, the discussion of Remark 4 applies to this example. This implies that the PPNG can be understood equivalently as the result of weighted $\mathcal{L}_{2}$-norm minimisation with $W(r)=r^{-m}$ as well as $\mathcal{L}_{p}$-norm minimisation with $W(r)=1$ for $m=\frac{1}{p-1}-1$.

The proposed work allows the exponent $p>1$ in the performance index to be a design parameter in addition to the choice of weighting function $W(r)$. Tuning of $p \in(1, \infty)$ will alter the distribution of control effort over the time interval. The expected behaviour can be understood by the physical meaning of the limiting cases, namely, the case with $p=1$ and $p=\infty$, while considering a constant weighting $W(r)=1$ used in the performance index to exclude the effect of weighting choice. In the case with $p=1$, the length of the time interval in which the control input remains large enough to suppress the error will be shortened at the cost of large initial command because minimisation of $\mathcal{L}_{1}$-norm of input has a tendency to reduce the duration of the interval with non-zero input. On the other hand, minimising $\mathcal{L}_{\infty}$-norm of input is to reduce the maximum value of input at the expense of longer duration of steering effort. Therefore, it is easy to reckon that the choice of $p$ will trade-off the uniformity of control effort against the magnitude of the peak input. However, care should be taken in that too small $p$ may lead to degraded robustness to process noise and too large $p$ may result in virtually changeless input history. 


\section{Discussions}

\section{Connections to Other Design Methodologies}

Relation between the proposed approach and Approach 1 of Sec. II.C is already discussed in Sec. III.B.1 through Example 1. The proposed linear non-quadratic design approach encompasses Approach 1, LQ optimal control, for the case with no state and state-input cross-coupling terms entering into the performance index, because the present study reduces to the LQ framework with the choice $p=2$.

The linear non-quadratic optimal guidance law also has relations to Approach 2, input linear parametrisation. The optimal solution of Eq. (34) is equivalent to the one derived by specifying the basis function of Approach 2 as

$$
\phi(\xi)=\left(\frac{\left|\Phi\left(\xi_{f}, \xi\right) b(\xi)\right|}{W(\xi)}\right)^{\frac{1}{p-1}}
$$

Equation (40) shows that the basis function is a non-negative function, i.e., $\phi(\xi) \geq 0$, regardless of $p$, because $W(\xi)>0$. The equality $\phi(\xi)=0$ holds for some $\xi$ in the open interval $\left(\xi_{0}, \xi_{f}\right)$ if and only if $W^{-1}(\xi)=0$, because $\Phi\left(\xi_{f}, \xi\right)>0$ and $b(\xi) \neq 0$ for all $\xi \in\left(\xi_{0}, \xi_{f}\right)$. Furthermore, at the final boundary $\xi_{f}$, the equality $\phi\left(\xi_{f}\right)=0$ holds if and only if $\left|b\left(\xi_{f}\right)\right| W^{-1}\left(\xi_{f}\right)=0$ since $b(\xi)$ is allowed to be zero at the boundary $\xi_{f}$.

Lastly, the proposed approach is equivalent to Approach 3, dynamic inversion, in which the desired closed-loop error dynamics is specified as

$$
e^{\prime}(\xi)+\left[\frac{\Phi\left(\xi_{f}, \xi\right) b(\xi)\left|\Phi\left(\xi_{f}, \xi\right) b(\xi)\right|^{\frac{1}{p-1}} W^{-\frac{1}{p-1}}(\xi)}{\int_{\xi}^{\xi_{f}} \Phi\left(\xi_{f}, \tau\right) b(\tau)\left|\Phi\left(\xi_{f}, \tau\right) b(\tau)\right|^{\frac{1}{p-1}} W^{-\frac{1}{p-1}}(\tau) d \tau}-a(\xi)\right] e(\xi)=0
$$

Note that Eq. (41) can be obtained by substituting Eq. (34) into the error dynamics equation in Eq. (24). Eq. (41) contains the fractional term $\frac{\Gamma(\xi)}{\int_{\xi}^{\xi_{f}} \Gamma(\tau) d \tau}$ where

$$
\Gamma(\xi) \triangleq \Phi\left(\xi_{f}, \xi\right) b(\xi)\left|\Phi\left(\xi_{f}, \xi\right) b(\xi)\right|^{\frac{1}{p-1}} W^{-\frac{1}{p-1}}(\xi)
$$

and therefore, it complies with the requirement for desired closed-loop error dynamics described in Remark 3.

In these regards, the new framework proposed is complementary or equivalent to existing design methodologies developed for finite-horizon error regulation each of which is based on the philosophy of optimal control (Approach 1), interpolation (Approach 2), and nonlinear control (Approach 3). The discussion implies that multiple design methodologies may lead to the same solution in guidance problems.

As discussed, the main potential advantage in practice is that the proposed approach allows us to consider some additional guidance objectives that were not able to be incorporated by having an additional degree-of-freedom. A 
follow-on study shall address the problem of how to co-design the weighting function $W(\xi)$ and the exponent $p$ to meet the necessities arise in practice since the proposed framework enables more generic optimal guidance.

\section{Properties of the Optimal Solution}

A remarkable point is that extending the class of performance index to allow non-quadratic input norms in formulation does not lead to any change in the linear structure of the feedback law, but only results in an altered structure of the gain. That is, the linear non-quadratic optimal guidance laws, as well as the LQ optimal guidance laws, have the form of linear error feedback while the difference resulting from cost functional type is the matter only of the gain $K^{*}(\xi)$.

The optimal gain $K^{*}(\xi)$ as defined in Eq. (34) does not change its sign during the engagement. As explained previously, the function $b(\xi)$ does not change its sign for $\xi \in\left(\xi_{0}, \xi_{f}\right)$ because $b(\xi) \neq 0$ in the interval by definition for controllability of the error dynamics. Also, we have $\operatorname{sgn}(\Gamma(\xi))=\operatorname{sgn}(b(\xi))$ from Eq. (42). These together lead to $\operatorname{sgn}\left(b(\xi) K^{*}(\xi)\right)=\operatorname{sgn}\left(\frac{\Gamma(\xi)}{\int_{\xi}^{\xi_{f}} \Gamma(\tau) d \tau}\right)=\operatorname{sgn}\left(\xi_{f}-\xi\right)=\operatorname{sgn}\left(\frac{d \xi}{d t}\right)$ while the independent variable $\xi$ is monotonic with respect to time $t$, showing invariance of $\operatorname{sgn}\left(K^{*}(\xi)\right)$. As a consequence, it follows from Eq. (41) that the convergence of error $e$ is monotonic with respect to the evolution of $\xi$, if the optimal guidance law Eq. (34) is employed and $a(\xi)=0$. Note here that $a(\xi)=0$ in most problems, given that the error dynamics is originally represented as Eq. (6) before introducing any assumption on the linearity of the error dynamics.

Another observation made is that the magnitude of the optimal gain $K^{*}(\xi)$ approaches infinity as $\xi \rightarrow \xi$. This ever-increasing property for the gain is essential for the final error to be zero rather than to have the error approaching exponentially to near zero.

It is desirable to prevent the guidance input from blowing up to infinity at any moment during the engagement, and at the final time in particular. Given that the proposed optimal guidance law is equivalent to Approach 2 as revealed in Sec. III.C.1, the condition $\phi(\xi)<\infty$ on the corresponding basis function given by Eq. (40) guarantees finiteness of the guidance command $u(\xi)$. Moreover, it is desirable in many guidance problems to have the final input being zero, i.e., $u\left(\xi_{f}\right)=0$, to ensure stable interception and to guarantee a sufficient margin in control authority to counteract with the potential undesirable errors near the end of engagement. The necessary and sufficient condition for $u\left(\xi_{f}\right)=0$ is $\phi\left(\xi_{f}\right)=0$. If the rate of guidance input is also desired to be zero at the final moment, $\phi^{\prime}\left(\xi_{f}\right)=0$ should be satisfied additionally. The functions $\Phi\left(\xi_{f}, \xi\right)$ and $b(\xi)$ are given by the guidance problem under consideration. Thus, the conditions described in terms of $\phi(\xi)$ should be satisfied by proper choices of the design parameters, namely, the weighting function $W(\xi)$ and the exponent $p$. 


\section{Numerical Simulation}

Numerical simulation is performed to support the theoretical findings of this study with the following objectives:

1) to verify the correctness of the analytic solution derived in this study (see Sec. IV.A)

2) to show the influences of the exponent $p$ on the command history and flight trajectory (see Sec. IV.B)

For these purposes, simulation is performed for the same problem formulation described in Sec. III.B.2, which presented PPNG as an illustrative example of the linear non-quadratic optimal error shaping guidance laws. Choosing the same PPNG example as the simulation scenario is to have better consistency of discussions with the preceding analyses. The simulation here concerns more about the outcome and validity of considering a non-traditional performance index type in optimal guidance problems than the potential superiority of a guidance law designed by utilising the proposed framework over a corresponding existing guidance law for a specific mission. Simulation for other problems such as linear non-quadratic optimal IACG or ITCG is omitted for this reason, though the proposed framework is generally applicable to various guidance problems.

The parameters used in the simulation are summarised in Table 1. The numerical simulation is performed using the 5th-order fixed-step solver in Simulink with the time step of 0.001[s].

Table 1 Simulation Parameters

\begin{tabular}{ccc}
\hline Parameter & Unit & Value \\
\hline Missile-target initial range, $r(0)$ & $\mathrm{km}$ & 10 \\
Missile speed, $V_{M}$ & $\mathrm{~m} / \mathrm{s}$ & 300 \\
Missile initial flight path angle, $\gamma_{M_{0}}$ & $\mathrm{deg}$ & 50 \\
Performance index type, $p$ & - & $1.1,1.25,1.5,2,4,5,10$ \\
Weighting function $W(r)$ & - & 1 \\
\hline
\end{tabular}

\section{A. Comparison Between Analytic and Numerical Solutions}

In the simulation, the analytic solution derived in Sec. III.B.2 is compared with the numerical solution obtained from trajectory optimisation for each $p$. The optimal guidance problem under consideration is a convex problem. Therefore, the problem is first discretised with step size of $\Delta r=0.5[\mathrm{~m}]$ which results in 20,000 nodes and then solved by the software CVX, a MATLAB package for modelling and solving convex programs [39, 40].

Fig. 2 shows the discrepancy between analytic solution $e_{\text {analytic }}(r)$ obtained from simulation of Eq. (39) and numerical solution $e_{\text {numerical }}(r)$ obtained by solving the discretised convex program where $e$ represents the ZEM $Z$. The absolute discrepancy shown in Fig. 2 is insignificant for every $p$ considering that the step size used in discretisation is $0.5[\mathrm{~m}]$ and the initial ZEM, $e\left(r_{0}\right)$, is in order of few kilometres. In this respect, Fig. 2 clearly shows that the analytic solution is almost identical to the corresponding numerical solution for each $p$, verifying that the analytic solution derived in this study is correct. 


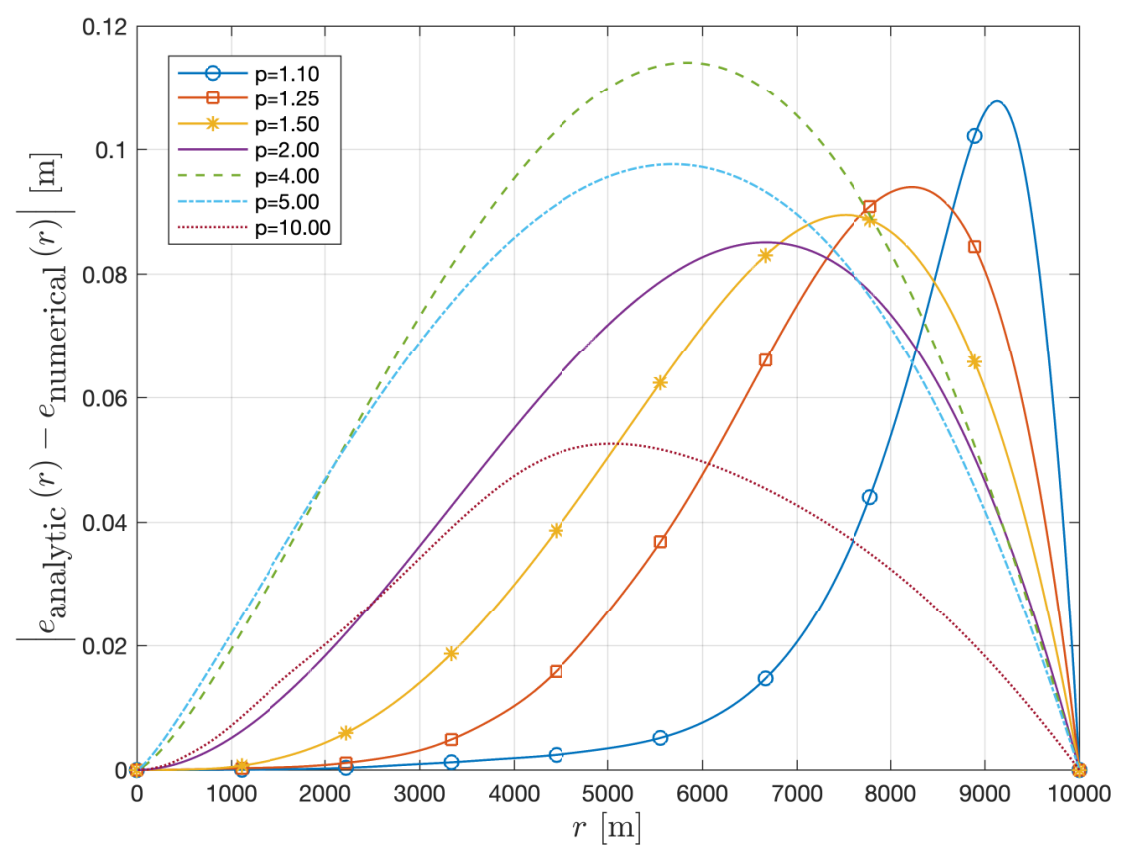

Fig. 2 Discrepancy Between Analytic and Numerical Solutions

\section{B. Influences of Exponent $p$ on Command and Trajectory}

In this section, the analytic solution derived in this study is simulated for each $p$ considered in Table 1 to show how the command history and the trajectories depend on the exponent $p$ in the performance index. Figs. 3-9 show the two-dimensional trajectory, range history, error history with respect to range and time, input history with respect to range and time, and optimal gain history, respectively.

Figs. 5-6 show that the rate of error convergence is faster with smaller $p$. The increased rate of error convergence comes at the cost of large initial input as shown in Figs. 7-8. Figs. 7-8 also demonstrate that the distribution of input over the duration of engagement becomes more uniform as $p$ increases. Moreover, Fig. 9 shows that the optimal gain $K^{*}$ approaches infinity as $r \rightarrow r_{f}=0$. All these observations made from the simulation results are consistent with the theoretical discussions in Secs. III.B.2 and III.C.2.

The effect of the type of input norm on the trajectory and command shown in the simulation results of Figs. 3-9 can be understood in the following manner. With the choice of weighting function given by $W(r)=1$, it follows from Eq. (39) that

$$
a_{M}^{n}(r)=\frac{r^{\frac{2 p-1}{p-1}}}{\int_{0}^{r} r^{\frac{p}{p-1}} d r} V_{M} \dot{\lambda}=\frac{2 p-1}{p-1} V_{M} \dot{\lambda} \triangleq N V_{M} \dot{\lambda}
$$

Considering the system dynamics explained in Sec. III.B.2 and Eqs. (1)-(2), the closed-loop error dynamics 
corresponding to Eq. (43) is given as $\frac{d e}{d r}=\frac{N}{r} e$. Consequently, the equations for the error $e=Z$, optimal gain $K^{*}$, and input $u=a_{M}^{n}$ can be obtained as functions of $r$ as

$$
\begin{aligned}
e(r) & =e\left(r_{0}\right) r^{N} \\
K^{*}(r) & =\frac{N V_{M}^{2}}{r^{2}} \\
u(r) & =-N V_{M}{ }^{2} r^{N-2} e\left(r_{0}\right)
\end{aligned}
$$

with $N$ as defined in Eq. (43). Eq. (43) shows that the linear non-quadratic optimal solution with $W(r)=1$ is simply the PPNG with the constant gain $N=2+\frac{1}{p-1}>2$. Note that $N=3$ if $p=2$, which accords with the well-known result on the optimality of PPNG with respect to the pure energy-type performance index. It is clear from Eq. (43) that $\frac{\partial N}{\partial p}<0$ for $p \in(1, \infty)$. Therefore, the smaller $p$ led to larger initial input and faster rate of error convergence in the simulation results. On the other hand, the command variability is reduced for larger $p$. This tendency is consistent with the physical meaning of $p$ expected in Sec. III.B.2 and the well-known behaviour of constant gain PPNG. In summary, the results clearly support the analytical discussions of the previous sections.

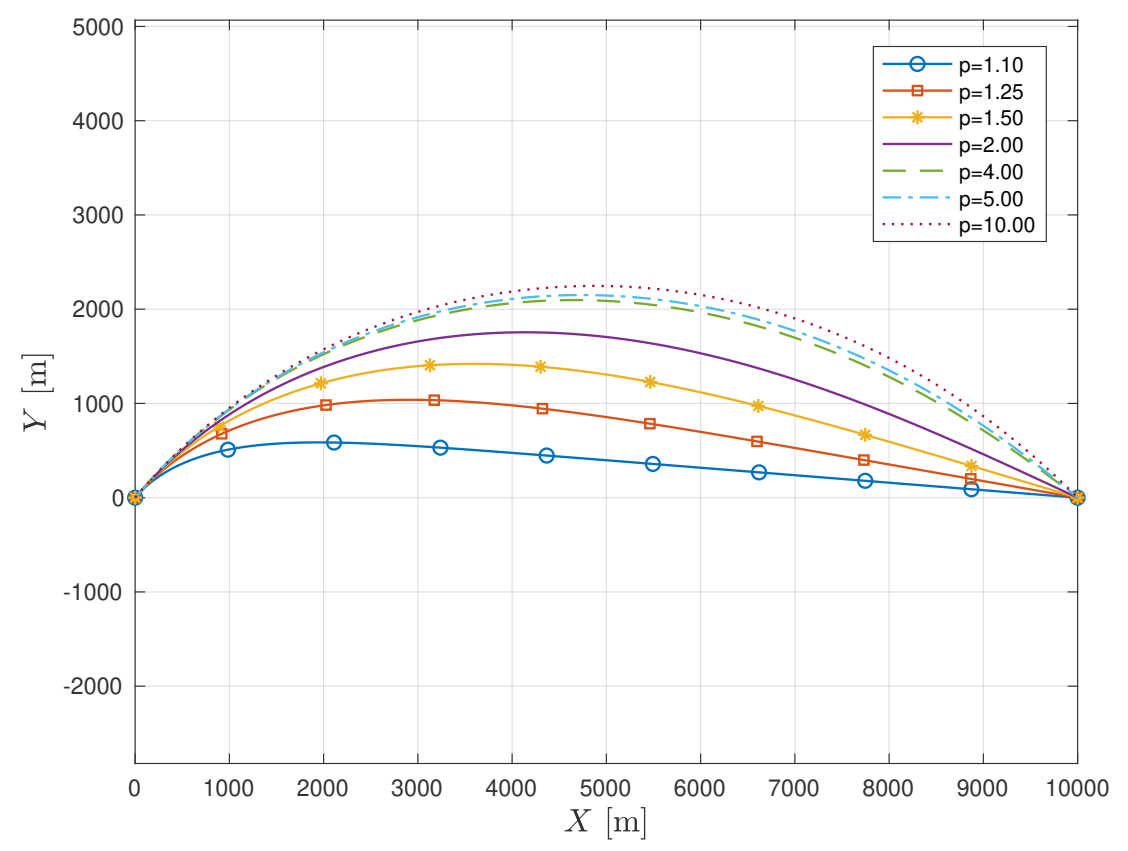

Fig. 3 Two-Dimensional Trajectory 


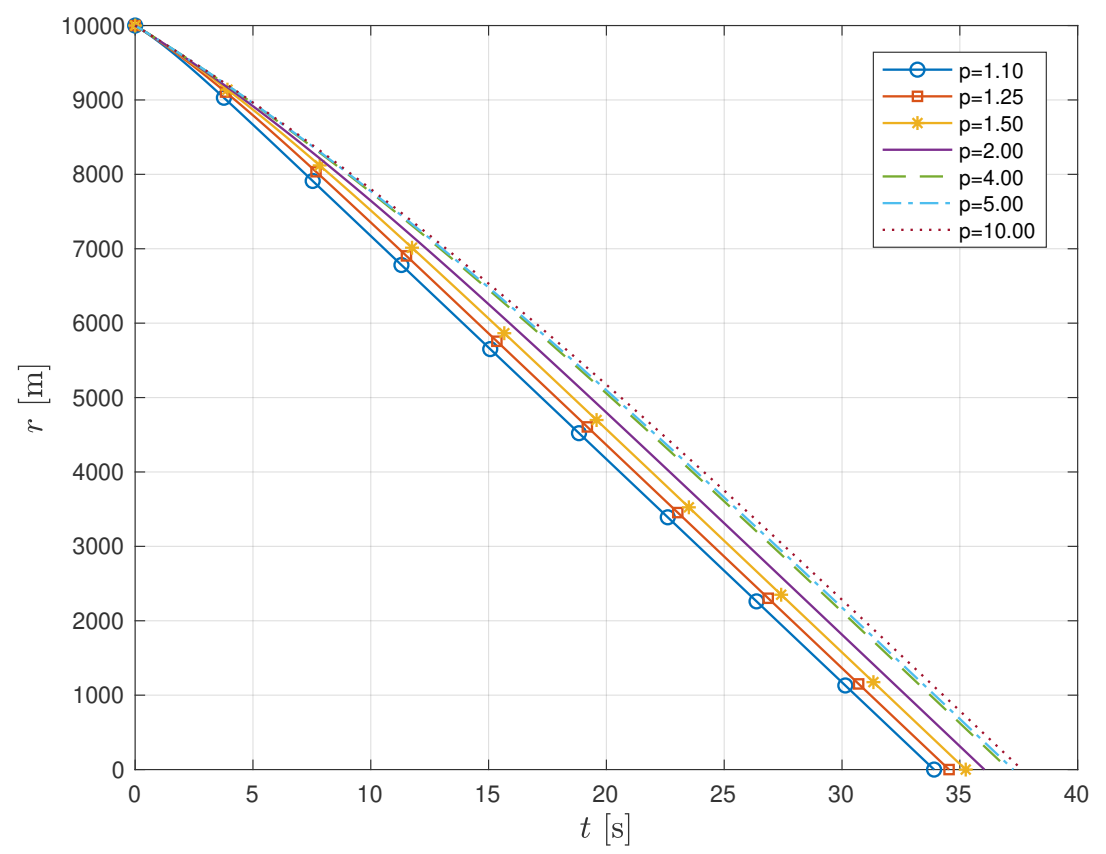

Fig. 4 Range History

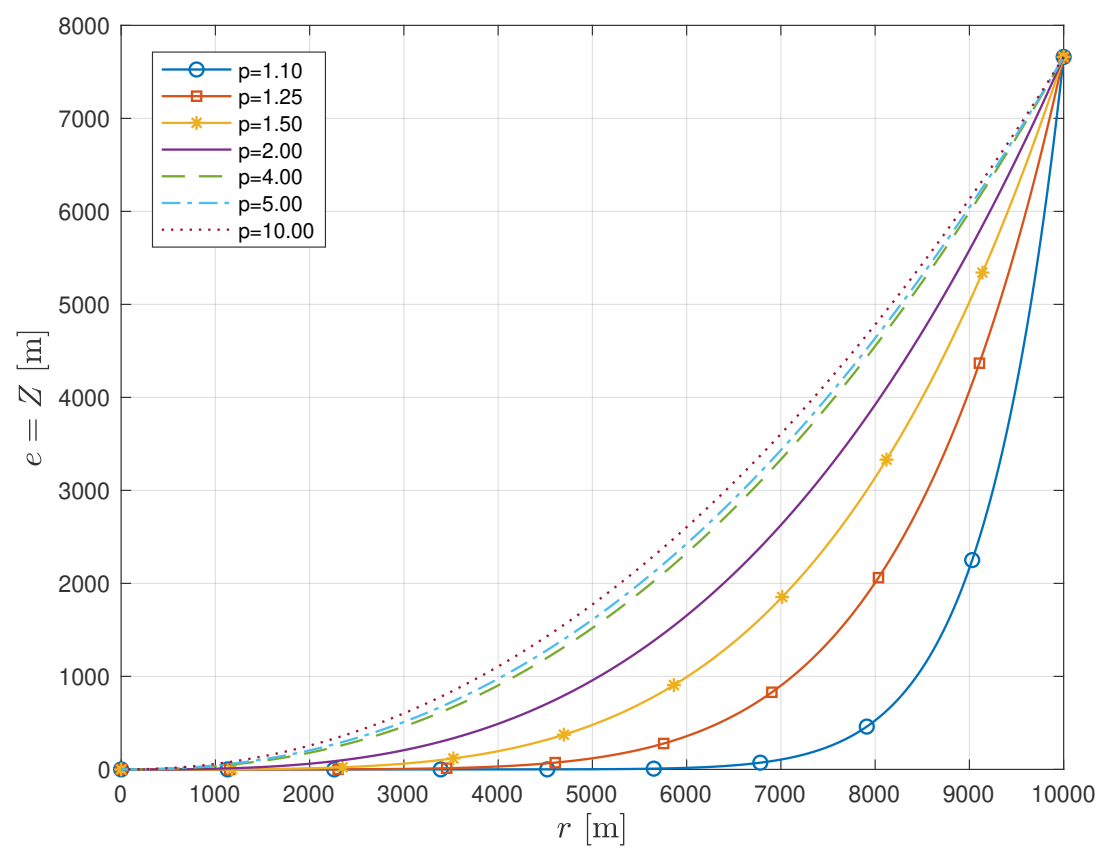

Fig. 5 Error (Zero Effort Miss) History with Respect to Range 


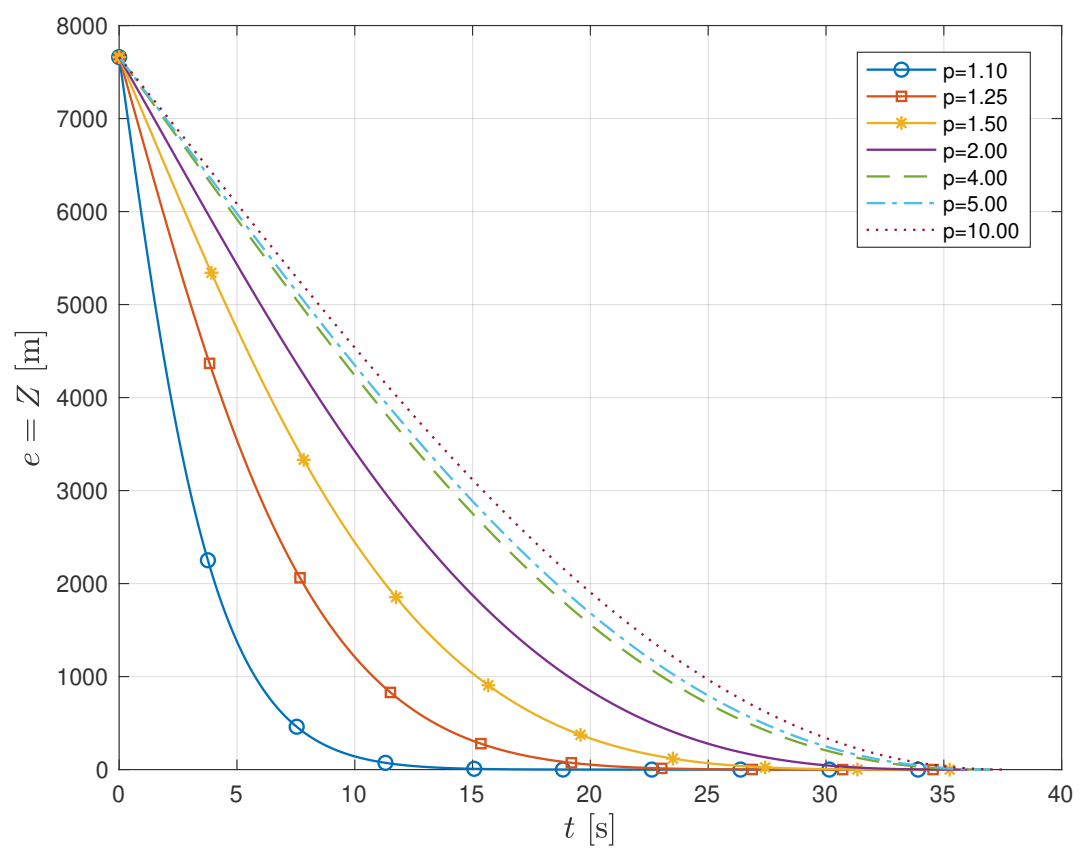

Fig. 6 Error (Zero Effort Miss) History with Respect to Time

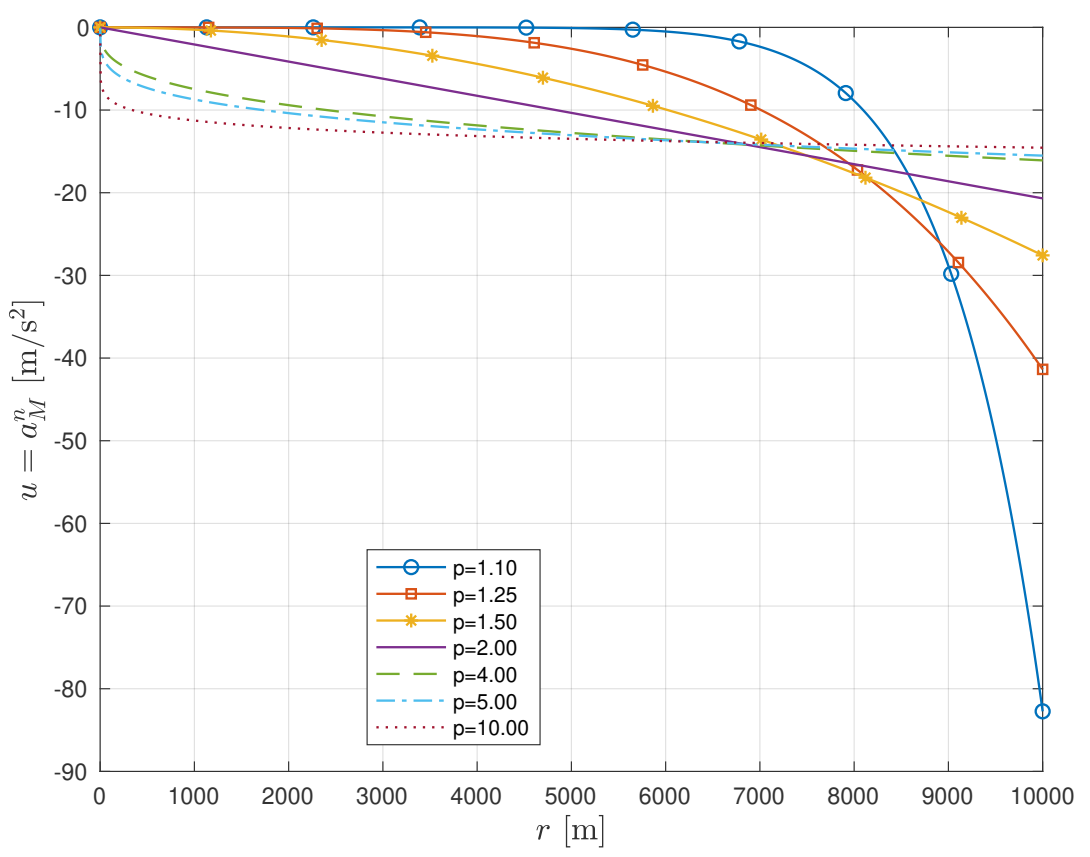

Fig. 7 Input (Lateral Acceleration) History with Respect to Range 


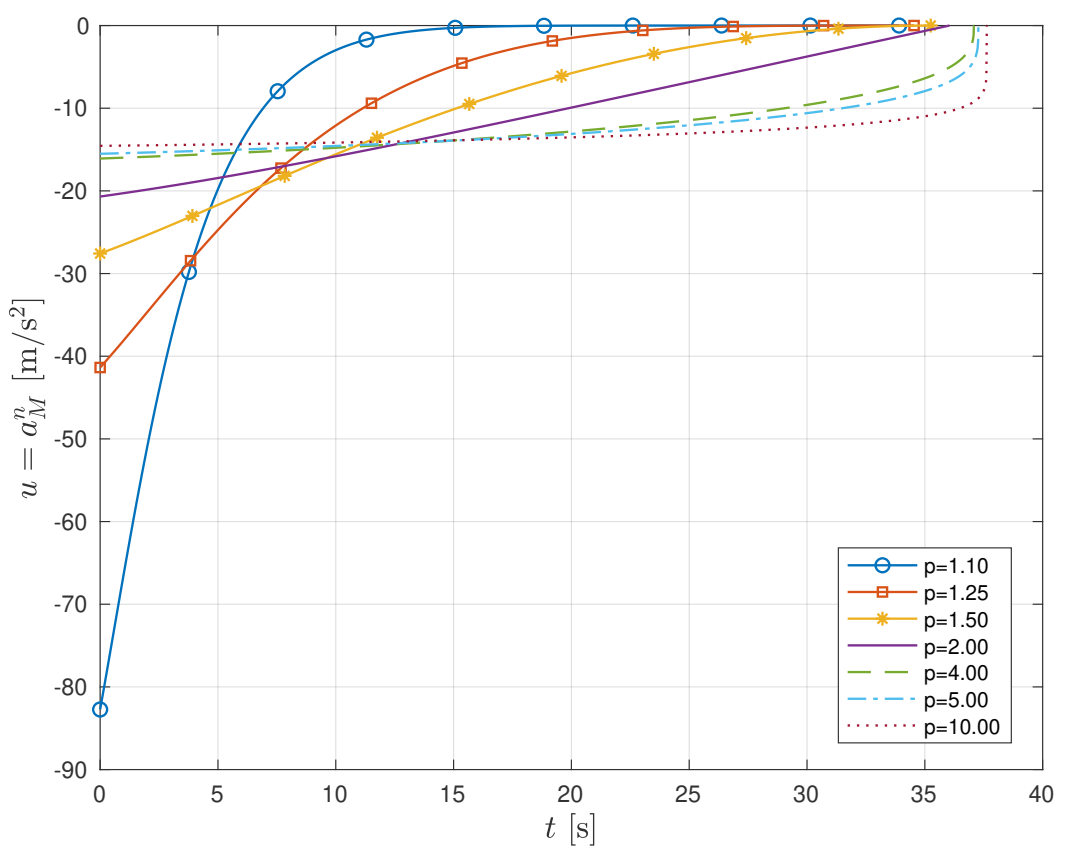

Fig. 8 Input (Lateral Acceleration) History with Respect to Time

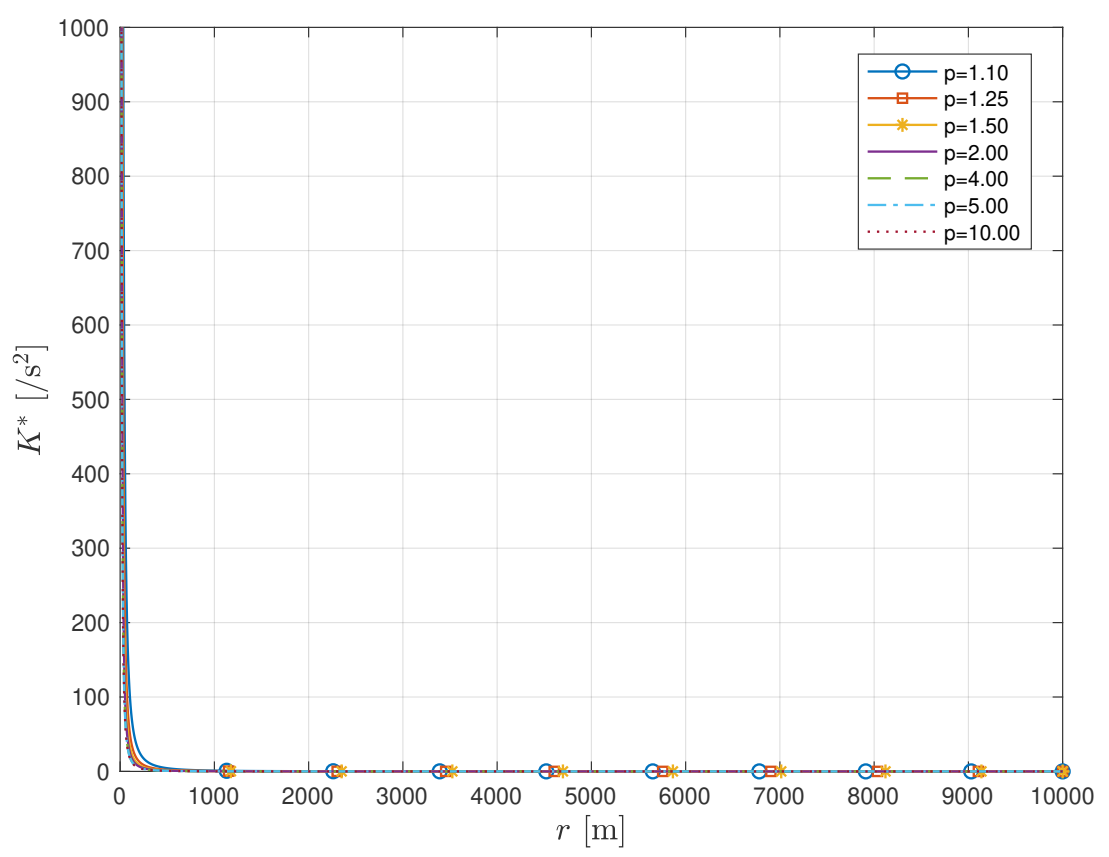

Fig. 9 Optimal Gain History 


\section{Conclusion}

The main question addressed in this study is the generalisation of the class of optimal missile guidance problems in terms of the type of performance index; from only quadratic cost to non-quadratic cost. This study presented the closed-form solution for optimal missile guidance problems with non-quadratic performance indices for the first time in the literature, to the best of our knowledge. The relation between the new linear non-quadratic optimal design framework and existing methods was also examined. It turned out that the proposed method has certain equivalence with the other optimal, interpolation, and nonlinear design philosophies.

The proposed framework can be utilised not only to extend existing guidance laws, but also to design new guidance laws for various objectives in a unified fashion as long as the given problem is formulated as a finite-horizon scalar error regulation problem. In particular, the proposed framework has potential significance in guidance problems in which minimisation of a quadratic performance index is not much physically meaningful or suitable. Unlike the earlier studies on optimal guidance laws, the proposed framework includes the type of input norm as a design parameter which can be chosen to meet various shaping necessities. The additional degree-of-freedom along with the weighting function yields more flexibility in the shaping of trajectory and command to meet the predetermined criteria of a specific mission. Tuning of the exponent in the non-quadratic input norm might be particularly useful for adjusting the uniformity of steering effort over time, as discussed in theoretical analyses and demonstrated by numerical simulation.

\section{Appendix}

\section{A. General First-Order Linear Nonhomogeneous Ordinary Differential Equation}

Let us consider the following first-order linear ordinary differential equation.

$$
\frac{d \psi}{d \xi}+p(\xi) \psi=r(\xi)
$$

We want to solve Eq. (A.1) for an interval $\xi \in[a, b]$ in which $r(\xi)$ is non-zero. Eq. (A.1) can be rewritten as

$$
(p(\xi) \psi-r(\xi)) d \xi+d \psi=0
$$

Let $F(\xi)$ be the integrating factor given as a function of $\xi$. Multiplying Eq. (A.2) by $F(\xi)$ yields

$$
F(\xi)(p(\xi) \psi-r(\xi)) d \xi+F(\xi) d \psi=0
$$


The following condition should hold with the integrating factor $F(\xi)$ for Eq. (A.3) to be an exact differential equation.

$$
\frac{\partial}{\partial \psi}[F(\xi)(p(\xi) \psi-r(\xi))]=\frac{\partial}{\partial \xi} F(\xi)
$$

Eq. (A.4) is a simple separable differential equation with respect to $\xi$, and the result is derived as

$$
F(\xi)=\exp \left(\int_{s_{0}}^{\xi} p(s) d s\right)
$$

Multiplying Eq. (A.1) by $F(\xi)$ obtained in Eq. (A.5), we have

$$
\exp \left(\int_{s_{0}}^{\xi} p(s) d s\right)\left[\frac{d \psi}{d \xi}+p(\xi) \psi\right]=\frac{d}{d \xi}\left[\exp \left(\int_{s_{0}}^{\xi} p(s) d s\right) \psi\right]=\exp \left(\int_{s_{0}}^{\xi} p(s) d s\right) r(\xi)
$$

Therefore, from Eq. (A.6), the general solution of Eq. (A.1) can be written as

$$
\psi(\xi)=\exp \left(-\int_{s_{0}}^{\xi} p(s) d s\right)\left[\int_{\tau_{0}}^{\xi} \exp \left(\int_{s_{0}}^{\tau} p(s) d s\right) r(\tau) d \tau+c\right]
$$

where $c$ is a constant. Eq. (A.7) can be understood as the invariance of the following quantity on the solution of Eq. (A.1).

$$
w(\xi, \psi) \triangleq \exp \left(\int_{s_{0}}^{\xi} p(s) d s\right) \psi(\xi)-\int_{\tau_{0}}^{\xi} \exp \left(\int_{s_{0}}^{\tau} p(s) d s\right) r(\tau) d \tau=c
$$

Note that $c$ can be determined by the initial condition.

\section{B. Hölder's Inequality}

A fundamental inequality of non-negative integrals referred to as Hölder's inequality is a useful tool for the mathematical analysis of $\mathcal{L}_{p}$ spaces. This inequality is one of the underlying principle leading to the proposed extension of the optimal guidance design method. The basic form of Hölder's inequality is summarised below.

Lemma 1 (Hölder's Inequality [41]).

Let

$$
\frac{1}{p}+\frac{1}{q}=1
$$

with $p, q>1$, and let $f(x)$ and $g(x)$ be $p$-th and $q$-th power integrable real functions, respectively. Then, Hölder's inequality for integrals states that

$$
\int_{a}^{b}|f(x) g(x)| d x \leq\left[\int_{a}^{b}|f(x)|^{p} d x\right]^{\frac{1}{p}}\left[\int_{a}^{b}|g(x)|^{q} d x\right]^{\frac{1}{q}}
$$


with equality holds when

$$
|g(x)|=\alpha|f(x)|^{p-1}
$$

for some $\alpha \neq 0$. If $p=q=2$, this inequality becomes Schwarz's inequality.

\section{Acknowledgements}

The authors would like to express gratitude to the colleagues and anonymous reviewers who have provided many meaningful suggestions that improved the quality of this study.

\section{References}

[1] Cho, H., Ryoo, C.-K., and Tahk, M.-J., “Closed-Form Optimal Guidance Law for Missiles of Time-Varying Velocity,” Journal of Guidance, Control, and Dynamics, Vol. 19, No. 5, 1996, pp. 1017-1022. doi:10.2514/3.21740

[2] Ryoo, C.-K., Cho, H., and Tahk, M.-J., “Optimal Guidance Laws with Terminal Impact Angle Constraint,” Journal of Guidance, Control, and Dynamics, Vol. 28, No. 4, 2005, pp. 724-732. doi:10.2514/1.8392

[3] Shaferman, V., and Shima, T., "Linear Quadratic Guidance Laws for Imposing a Terminal Intercept Angle," Journal of Guidance, Control, and Dynamics, Vol. 31, No. 5, 2008, pp. 1400-1412. doi:10.2514/1.32836

[4] Reisner, D., and Shima, T., "Optimal Guidance-to-Collision Law for an Accelerating Exoatmospheric Interceptor Missile,” Journal of Guidance, Control, and Dynamics, Vol. 36, No. 6, 2013, pp. 1695-1708. doi:10.2514/1.61258

[5] Cho, H., Ryoo, C.-K., Tsourdos, A., and White, B., "Optimal Impact Angle Control Guidance Law Based on Linearization About Collision Triangle," Journal of Guidance, Control, and Dynamics, Vol. 37, No. 3, 2014, pp. 958-964. doi:10.2514/1.62910

[6] Weiss, M., and Shima, T., "Minimum Effort Pursuit/Evasion Guidance with Specified Miss Distance," Journal of Guidance, Control, and Dynamics, Vol. 39, No. 5, 2016, pp. 1069-1079. doi:10.2514/1.G001623

[7] Lee, C.-H., Optimal Guidance Laws Using Generalized Weighting Functions, Ph.D. Dissertation, Department of Aerospace Engineering, Korea Advanced Institute of Science and Technology, Daejeon, Korea, 2013.

[8] Lee, J.-I., Jeon, I.-S., and Lee, C.-H., “Command-Shaping Guidance Law Based on a Gaussian Weighting Function,” IEEE Transactions on Aerospace and Electronic Systems, Vol. 50, No. 1, 2014, pp. 772-777. doi:10.1109/TAES.2013.120353

[9] Weiss, M., and Shima, T., "Optimal Linear-Quadratic Missile Guidance Laws with Penalty on Command Variability," Journal of Guidance, Control, and Dynamics, Vol. 38, No. 2, 2015, pp. 226-237. doi:10.2514/1.G000738

[10] Ryu, M.-Y., Lee, C.-H., and Tahk, M.-J., "Command Shaping Optimal Guidance Laws Against High-Speed Incoming Targets," Journal of Guidance, Control, and Dynamics, Vol. 38, No. 10, 2015, pp. 2025-2033. doi:10.2514/1.G001131

[11] Levy, M., Shima, T., and Gutman, S., "Linear Quadratic Integrated Versus Separated Autopilot-Guidance Design,” Journal of Guidance, Control, and Dynamics, Vol. 36, No. 6, 2013, pp. 1722-1730. doi:10.2514/1.61363 
[12] Levy, M., Shima, T., and Gutman, S., "Single Versus Two-Loop Full-State Multi-Input Missile Guidance," Journal of Guidance, Control, and Dynamics, Vol. 38, No. 5, 2015, pp. 843-853. doi:10.2514/1.G000171

[13] Kim, J.-H., Whang, I. H., and Kim, B. M., "Finite Horizon Integrated Guidance and Control for Terminal Homing in Vertical Plane," Journal of Guidance, Control, and Dynamics, Vol. 39, No. 5, 2016, pp. 1104-1112. doi:10.2514/1.G001699

[14] Lee, C.-H., Tahk, M.-J., and Lee, J.-I., "Generalized Formulation of Weighted Optimal Guidance Laws with Impact Angle Constraint," IEEE Transactions on Aerospace and Electronic Systems, Vol. 49, No. 2, 2013, pp. 1317-1322. doi:10.1109/TAES.2013.6494416

[15] Shneydor, N. A., Missile Guidance and Pursuit: Kinematics, Dynamics and Control, Horwood Publishing, Chichester, West Sussex, United Kingdom, 1998, pp. 196-197.

[16] Jeon, I.-S., and Lee, J.-I., “Optimality of Proportional Navigation Based on Nonlinear Formulation,” IEEE Transactions on Aerospace and Electronic Systems, Vol. 46, No. 4, 2010, pp. 2051-2055. doi:10.1109/TAES.2010.5595614

[17] Lee, Y.-I., Kim, S.-H., and Tahk, M.-J., “Optimality of Linear Time-Varying Guidance for Impact Angle Control,” IEEE Transactions on Aerospace and Electronic Systems, Vol. 48, No. 4, 2012, pp. 2802-2817. doi:10.1109/TAES.2012.6324662

[18] Bass, R. W., and Webber, R. F., "Optimal Nonlinear Feedback Control Derived from Quartic and Higher-Order Performance Criteria," IEEE Transactions on Automatic Control, Vol. 11, No. 3, 1966, pp. 448-454. doi:10.1109/TAC.1966.1098381

[19] Asseo, S. J., "Optimal Control of a Servo Derived from Nonquadratic Performance Criteria," IEEE Transactions on Automatic Control, Vol. 14, No. 4, 1969, pp. 404-407. doi:10.1109/TAC.1969.1099200

[20] Bernstein, D. S., "Nonquadratic Cost and Nonlinear Feedback Control," International Journal of Robust and Nonlinear Control, Vol. 3, No. 3, 1993, pp. 211-229. doi:10.1002/rnc.4590030303

[21] Vidyasagar, M., and Mueller, G. S., "Optimal Control of Linear Systems for a Class of Non-quadratic, Convex Functionals," International Journal of Control, Vol. 19, No. 3, 1974, pp. 657-660. doi:10.1080/00207177408932661

[22] Shamma, J. S., and Xiong, D., "Linear Nonquadratic Optimal Control," IEEE Transactions on Automatic Control, Vol. 42, No. 6, 1997, pp. 875-879. doi:10.1109/9.587349

[23] You, Y., "Synthesis of Time-Variant Optimal Control with Nonquadratic Criteria," Journal of Mathematical Analysis and Applications, Vol. 209, No. 2, 1997, pp. 662-682. doi:10.1006/jmaa.1997.5399

[24] Pan, L. P., and Teo, K. L., "Linear-Nonquadratic Optimal Control Problems with Terminal Inequality Constraints,” Journal of Mathematical Analysis and Applications, Vol. 212, No. 1, 1997, pp. 176-189. doi:10.1006/jmaa.1997.5489

[25] Le Breton, A., "A Note on the Synthesis of Nonquadratic Optimal Control in a One-Dimensional Linear System," Journal of Mathematical Analysis and Applications, Vol. 243, No. 1, 2000, pp. 32-46. doi:10.1006/jmaa.1999.6645 
[26] Hsu, C.-H., and Shamma, J. S., "Further Results on Linear Nonquadratic Optimal Control," IEEE Transactions on Automatic Control, Vol. 46, No. 5, 2001, pp. 732-736. doi:10.1109/9.920791

[27] Rockafellar, R. T., and Goebel, R., Linear-Convex Control and Duality, World Scientific Publishing Co. Pte. Ltd., Singapore, 2008, pp. 280-299. doi:10.1142/9789812776075_0017

[28] Heller, C., Yaesh, I., Ben-Asher, J., and Rusnak, I., "Lq-Optimal Missile Guidance and its Relation to Mixed L2/Lq Laws," AIAA Guidance, Navigation, and Control Conference, Chicago, IL, 2009. doi:10.2514/6.2009-5895

[29] Lee, C.-H., Shin, H.-S., Lee, J.-I., and Tahk, M.-J., “Zero-Effort-Miss Shaping Guidance Laws,” IEEE Transactions on Aerospace and Electronic Systems, Vol. 54, No. 2, 2018, pp. 693-705. doi:10.1109/TAES.2017.2764258

[30] He, S., and Lee, C.-H., "Optimality of Error Dynamics in Missile Guidance Problems," Journal of Guidance, Control, and Dynamics, Vol. 41, No. 7, 2018, pp. 1624-1633. doi:10.2514/1.G003343

[31] Seo, M.-G., Lee, C.-H., and Tahk, M.-J., "New Design Methodology for Impact Angle Control Guidance for Various Missile and Target Motions," IEEE Transactions on Control Systems Technology, Vol. 26, No. 6, 2018, pp. $2190-2197$. doi:10.1109/TCST.2017.2749560

[32] He, S., and Lee, C.-H., “Gravity-Turn-Assisted Optimal Guidance Law,” Journal of Guidance, Control, and Dynamics, Vol. 41, No. 1, 2018, pp. 171-183. doi:10.2514/1.G002949

[33] Tahk, M.-J., Shim, S.-W., Hong, S.-M., Choi, H.-L., and Lee, C.-H., "Impact Time Control Based on Time-to-Go Prediction for Sea-Skimming Antiship Missiles,” IEEE Transactions on Aerospace and Electronic Systems, Vol. 54, No. 4, 2018, pp. 2043-2052. doi:10.1109/TAES.2018.2803538

[34] Bryson, Jr., A. E., and Ho, Y.-C., Applied Optimal Control: Optimization, Estimation, and Control, Hemisphere Publishing Corporation, Washington, D.C., 1975, pp. 158-167.

[35] Shin, H.-S., Study on Cooperative Missile Guidance for Area Air Defence, Ph.D. Dissertation, Department of Informatics \& Systems Engineering, Autonomous Systems Group, Cranfield University, Cranfield, Bedfordshire, UK, 2010.

[36] Zarchan, P., Tactical and Strategic Missile Guidance, American Institute of Aeronautics and Astronautics, Reston, VA, 2012, pp. 569-601.

[37] Lin, C.-F., Modern Navigation, Guidance, and Control Processing, Volume II, Prentice Hall, Englewood Cliffs, NJ, 1991, pp. 562-583.

[38] Cho, N., Kim, Y., and Shin, H.-S., "Generalization of Linearly Parametrized Trajectory Shaping Guidance Laws,” 20th IFAC World Congress, Toulouse, France, 2017. doi:10.1016/j.ifacol.2017.08.1403

[39] Grant, M., and Boyd, S., “CVX: MATLAB Software for Disciplined Convex Programming, version 2.1," http: //cvxr . com/ cvx, August 2019. 
[40] Grant, M., and Boyd, S., "Graph Implementations for Nonsmooth Convex Programs," Recent Advances in Learning and Control, edited by V. Blondel, S. Boyd, and H. Kimura, Lecture Notes in Control and Information Sciences, Springer, London, UK, 2008, pp. 95-110. doi:10.1007/978-1-84800-155-8_7

[41] Beckenbach, E. F., and Bellman, R., Inequalities, Springer-Verlag, Berlin, Germany, 1961, pp. 19-23. 
2019-12-29

Generalized formulation of linear nonquadratic weighted optimal error shaping guidance laws

\author{
Cho, Namhoon
}

AIAA

Che N, Kim Y, Shin H-S. (2020)Generalized formulation of linear nonquadratic weighted optimal error shaping guidance laws. Journal of Guidance, Control, and Dynamics, Volume 43, Issue 6, June 2020, pp. 1143-1153

https://doi.org/10.2514/1.G004813

Downloaded from Cranfield Library Services E-Repository 